typeset using JPSJ.sty $<$ ver.1.0b $>$

\title{
Strong Coupling Approach to the $d-p$ Model on the Basis of Fermi Liquid Theory
}

\author{
Shigeru KoIKegam \\ Department of Physics, Kyoto University, Kyoto 606-8224
}

(Received December 10, 1997 )

\begin{abstract}
We study the superconducting transition temperature $T_{\mathrm{c}}$ of the bilayer $d-p$ model with $d_{x^{2}-y^{2-}}$ wavelike attractive interaction based on the formalism first employed by Nozières and SchmittRink. In the strong coupling regime, $T_{\mathrm{c}}$ obtained through this formalism are much suppressed, compared with those through Thouless criterion only. We also find that, whether the interlayer coupling exsists or not, $T_{\mathrm{c}}$ is almost propotional to the Fermi energy $E_{\mathrm{F}}$ in the strong coupling regime. Thus, we can reproduce the essential nature in the underdoped region.
\end{abstract}

KEYWORDS: high- $T_{c}$ superconductor, bilayer $d$ - $p$ model, Nozières-Schmitt-Rink formalism, Thouless criterion

The pseudogap state of underdoped cuprates has been a very important issue in the study of high- $T_{\mathrm{c}}$ superconductors (HTSC). Since the discovery of HTSC, the presence of an excitation gap, both in the charge and spin dynamics of underdoped cuprates in the normal state, has been indicated in several different experiments. The normal state transport properties (in-plane resistivity, Hall effect) and static susceptibility change their temperature dependences below a characteristic temperature $T^{*} .1$ The electronic specific heat analysis has shown that the electronic entropy starts to decrease at the temperature well above $T_{\mathrm{c}}$.2) The nuclear magnetic resonance (NMR) experiments have found the anomalous temperature dependences both of Knight shift and relaxation rate in almost all kinds of HTSC 3.4) The suppression of the in-plane scattering rate well above $T_{\mathrm{c}}$ has been derived from the optical conductivity mesurement.5)

From all these various experimental results, we can deduce the existence of some kind of electronic bound states in the normal state of underdoped cuprates, whose characteristic energy should be related to $T^{*}$. In order to explain this bound state formation, the theoretical works that identify $T^{*}$ with the singlet resonating-valence-bond (RVB) formation temperture have been carried out earlier.6) In the recent years, angle resolved photo-emission spectroscopy (ARPES) has revealed the electronic structure of a pseudogap in the normal state, which has a highly anistropic momentum dependence, $d_{x^{2}-y^{2}}$, as well as in the superconducting state. 68

Based on this result, it may be natural that the origin of the pseudogap is in the preformed Cooper pair which cannot Bose-condensate due to its thermal fluctuation. The thermal fluctuation of the Cooper pair is neglected to determine $T_{\mathrm{c}}$ in the original BCS theory, which is suitable for the description of the weak coupling regime where the electronic Fermi temperature $T_{\mathrm{F}}$ is much larger than the temperature $T$ and the superconducting carrier density is small compared to the degen-

*E-mail: koike@ton.scphys.kyoto-u.ac.jp erated electronic one. However, in underdoped cuprates, which are believed to be in the strong coupling regime where the electronic Fermi energy $E_{\mathrm{F}}$ is much reduced by the strong correlation, the thermal fluctuation cannot be neglected over the wide temperature range above $T_{\mathrm{c}}$, where $T$ may exceed the Fermi temperature $T_{\mathrm{F}}$.

For the above reason, we use the formalism first employed by Nozières and Schmitt-Rink (NSR), 9 ) in order to estimate $T_{\mathrm{c}}$ of the bilayer $d$ - $p$ model in such a strong coupling regime. Their formalism and continuum models introduced in Ref. 9 have been adopted by the several authors in the past in order to study the 2 r dimensional strong coupling superconductivity 10 , 11 , 22 The model Hamiltonian is as follows:

$$
\begin{aligned}
H= & H_{+}+H_{-}+H_{d} \\
& -\mu \sum_{\boldsymbol{k}_{\sigma \tau}}\left(d_{\boldsymbol{k}_{\sigma \tau}}^{\dagger} d_{\boldsymbol{k}_{\sigma \tau}}+p_{\boldsymbol{k}_{\sigma \tau}}^{x \dagger} p_{\boldsymbol{k}_{\sigma \tau}}^{x}+p_{\boldsymbol{k}_{\sigma \tau}}^{y \dagger} p_{\boldsymbol{k}_{\sigma \tau}}^{y}\right) .
\end{aligned}
$$

Here $d_{\boldsymbol{k}_{\sigma \tau}}\left(d_{\boldsymbol{k}_{\sigma \tau}}^{\dagger}\right)$ and $p_{\boldsymbol{k}_{\sigma \tau}(y)}^{(x)}\left(p_{\boldsymbol{k}_{\sigma \tau}(y) \dagger}^{(y)}\right)$ are the annihilation (creation) operator for $d$ - and $p^{x(y)}$-electron of momentum $\boldsymbol{k}$, spin $\sigma=\{\uparrow, \downarrow\}$ and layer $\tau=\{+,-\}$, respectively. $\mu$ is the chemical potential. The non-interacting part $H_{ \pm}$is represented by

$$
\begin{aligned}
& H_{ \pm}=\sum_{\boldsymbol{k}_{\sigma}}\left(\begin{array}{ccc}
d_{\boldsymbol{k}_{\sigma \pm}^{\dagger}}^{\dagger} & p_{\boldsymbol{k}_{\sigma \pm} x^{\dagger}} & p_{\boldsymbol{k}_{\sigma \pm}}^{y \dagger}
\end{array}\right) \\
& \times\left(\begin{array}{ccc}
\varepsilon_{d} \mp t_{z} & \zeta_{\boldsymbol{k}}^{x} & \zeta_{\boldsymbol{k}}^{y} \\
-\zeta_{\boldsymbol{k}}^{x} & \varepsilon_{p} & \zeta_{\boldsymbol{k}}^{p} \\
-\zeta_{\boldsymbol{k}}^{y} & \zeta_{\boldsymbol{k}}^{p} & \varepsilon_{p}
\end{array}\right)\left(\begin{array}{c}
d_{\boldsymbol{k}_{\sigma \pm}} \\
p_{\boldsymbol{k}_{\sigma \pm}^{x}}^{x} \\
p_{\boldsymbol{k}_{\sigma \pm}}^{y}
\end{array}\right),
\end{aligned}
$$

where $\zeta_{\boldsymbol{k}}^{x(y)}=2 \mathrm{i} t \sin \frac{k_{x(y)}}{2}$ and $\zeta_{\boldsymbol{k}}^{p}=-4 t_{p p} \sin \frac{k_{x}}{2} \sin \frac{k_{y}}{2}$. We introduce the three types of transfer energies, $t, t_{p p}$ and $t_{z}$. The first two are the intralayer transfer energies for the nearest neighbors between $d$ - and $p^{x(y)}$-orbitals and between $p^{x}$ - and $p^{y}$-orbitals, respectively. The third is the interlayer transfer energy for between $d$-orbitals. In the following part of this letter, we take $t$ as the unit of energy. The residual term $H_{d}$ in eq. (1) represents the attractive interaction between intralayer $d$-orbitals, 
described as follows:

$$
\begin{aligned}
H_{d}=-\frac{U_{d}}{N} \sum_{\boldsymbol{k} \boldsymbol{k}^{\prime}} & g_{\boldsymbol{k}} g_{\boldsymbol{k}^{\prime}} \\
& \times \sum_{\boldsymbol{q}_{\tau}} d_{\boldsymbol{k} \uparrow \tau}^{\dagger} d_{\boldsymbol{q}-\boldsymbol{k}_{\downarrow \tau}}^{\dagger} d_{\boldsymbol{q}-\boldsymbol{k}_{\downarrow}^{\prime}{ }^{\prime}} d_{\boldsymbol{k}^{\prime} \uparrow \tau},
\end{aligned}
$$

where $g_{\boldsymbol{k}}=\cos k_{x}-\cos k_{y}$ is the $d_{x^{2}-y^{2} \text {-wavelike form }}$ factor and $N$ is the number of $\mathrm{Cu}$ sites.

Then, we calculate the pairing correlation function for $d$-electrons Diagonalizing $H_{+}+H_{-}$by the unitary transformation,13) we can obtain the band dispersion, $\varepsilon_{\boldsymbol{k}_{ \pm}}^{\alpha}$, and its weight for the $d$-electron, $z_{\boldsymbol{k}_{+}}^{\alpha}$, where $\alpha=\{0, \pm 1\}$ is the band index. They are defined by

$$
\varepsilon_{\boldsymbol{k}_{ \pm}}^{\alpha}=\frac{2}{\sqrt{3}}\left|t_{\boldsymbol{k}_{ \pm}}\right| \cos \left(\frac{\varphi_{\boldsymbol{k}_{ \pm}}}{3}+\frac{2}{3} \pi \alpha\right)+\frac{\Delta_{ \pm}}{3},
$$

with

$$
\begin{aligned}
t_{\boldsymbol{k}_{ \pm}}^{2}= & \frac{\Delta_{ \pm}^{2}}{3}+\left(\zeta_{\boldsymbol{k}}^{p}\right)^{2}+\left|\zeta_{\boldsymbol{k}}^{x}\right|^{2}+\left|\zeta_{\boldsymbol{k}}^{y}\right|^{2} \\
\varphi_{\boldsymbol{k}_{ \pm}}= & \frac{\pi}{2}+\left(\frac{\pi}{2}-\phi_{\boldsymbol{k}_{ \pm}}\right) \operatorname{sgn}\left(s_{\boldsymbol{k}_{ \pm}^{3}}^{3}\right) \\
s_{\boldsymbol{k}_{ \pm}}^{3}= & \frac{\Delta_{ \pm}^{3}}{27}+\Delta_{ \pm}\left(\left(\zeta_{\boldsymbol{k}}^{p}\right)^{2}-\frac{t_{\boldsymbol{k}_{ \pm}}^{2}}{3}\right) \\
& \quad-\zeta_{\boldsymbol{k}}^{p}\left(\zeta_{\boldsymbol{k}}^{x} \zeta_{\boldsymbol{k}}^{y *}+\zeta_{\boldsymbol{k}}^{x *} \zeta_{\boldsymbol{k}}^{y}\right) \\
\phi_{\boldsymbol{k}_{ \pm}=} & \arctan \left(\left|s_{\boldsymbol{k}_{ \pm}}^{6}-\frac{4}{27} t_{\boldsymbol{k}_{ \pm}^{6}}^{6}\right|^{1 / 2} /\left|s_{\boldsymbol{k}_{ \pm}}^{3}\right|\right), \\
\Delta_{ \pm}= & \varepsilon_{d} \mp t_{z}-\varepsilon_{p}
\end{aligned}
$$

and

$$
\begin{aligned}
z_{\boldsymbol{k}_{ \pm}}^{\alpha} & =\frac{\left(\zeta_{\boldsymbol{k}}^{p}-\varepsilon_{\boldsymbol{k}_{ \pm}}^{\alpha}\right)\left(\zeta_{\boldsymbol{k}}^{p}+\varepsilon_{\boldsymbol{k}_{ \pm}}^{\alpha}\right)}{\left(\varepsilon_{\boldsymbol{k}_{ \pm}}^{\beta}-\varepsilon_{\boldsymbol{k}_{ \pm}}^{\alpha}\right)\left(\varepsilon_{\boldsymbol{k}_{ \pm}^{\alpha}}^{\alpha}-\varepsilon_{\boldsymbol{k}_{ \pm}}^{\gamma}\right)}, \\
\alpha & \neq \beta, \quad \beta \neq \gamma, \quad \gamma \neq \alpha .
\end{aligned}
$$

Using these expressions, the intra-(inter-)layer pairing correlation function, $\chi_{+(-)}^{d d}$, is represented by

$$
\begin{aligned}
& \chi_{ \pm}^{d d}(\boldsymbol{q}, \omega) \\
& =\frac{U_{d}}{2 N_{\mathrm{L}}} \sum_{\boldsymbol{k}} g_{\boldsymbol{k}}^{2} \sum_{\alpha \beta \tau} \\
& \quad \times \frac{z_{\boldsymbol{k}_{\tau}}^{\alpha} z_{\boldsymbol{q}-\boldsymbol{k}_{ \pm \tau}}^{\beta}\left\{1-f\left(E_{\boldsymbol{k}_{\tau}}^{\alpha}\right)-f\left(E_{\boldsymbol{q}_{-} \boldsymbol{k}_{ \pm \tau}}^{\beta}\right)\right\}}{E_{\boldsymbol{k}_{\tau}}^{\alpha}+E_{\boldsymbol{q}-\boldsymbol{k}_{ \pm \tau}}^{\beta}-\omega}
\end{aligned}
$$

Here $E_{\boldsymbol{k}_{ \pm}}^{\alpha}=\varepsilon_{\boldsymbol{k}_{+}}^{\alpha}-\mu$, and $f(\omega)$ represents the conventional Fermi distribution function. $N_{\mathrm{L}}$ is the number of $\boldsymbol{k}$-space lattice points in the first Brillouin zone (FBZ).

NSR formalism takes into account the contribution of the phase shift from the scattering $t$-matrix to the thermodynamic potential, which leads to the equation that determines the critical temperature $T_{\mathrm{c}}^{\mathrm{NSR}}$ as the Bose-Einstein condensation temperature for the density of pairs $n-n_{\mathrm{f}}$, where $n$ is the total Fermion density and $n_{\mathrm{f}}$ is the free part. First, the condition for the Cooper instability is given by

$$
0=1-\chi_{+}^{d d}(0,0)
$$

EPS File fig1.ps not found

Fig. 1. $T_{\mathrm{c}}^{\mathrm{MF}}$ and $T_{\mathrm{c}}^{\mathrm{NSR}}$ as functions of total electron number $n$ in (a) for $t_{z}=0.00 t$ and in (b) for $t_{z}=0.20 t$. In both panels the closed symbols denote the results for $T_{\mathrm{c}}^{\mathrm{MF}}$ and the open ones for $T_{\mathrm{c}}^{\mathrm{NSR}}$. The circles, squares and diamonds correspond to $\varepsilon_{d}=0.40 t, 0.80 t$ and $1.20 t$, respectively. The insets show the corresponding ratios of $d$-hole number $n_{\mathrm{h}}^{d}$ to $p$-hole number $n_{\mathrm{h}}^{p}$ for every $\varepsilon_{d}$ as functions of $n$. The correspondences of the symbols to $\varepsilon_{d}$ are the same.

$$
\begin{aligned}
=1-\frac{U_{d}}{2 N_{\mathrm{L}}} & \sum_{\boldsymbol{k}} g_{\boldsymbol{k}}^{2} \sum_{\alpha \beta \tau} \\
& \times \frac{z_{\boldsymbol{k}_{\tau}}^{\alpha} z_{\boldsymbol{k}_{\tau}}^{\beta}\left\{1-f\left(E_{\boldsymbol{k}_{\tau}}^{\alpha}\right)-f\left(E_{\boldsymbol{k}_{\tau}}^{\beta}\right)\right\}}{E_{\boldsymbol{k}_{\tau}}^{\alpha}+E_{\boldsymbol{k}_{\tau}}^{\beta}},
\end{aligned}
$$

and the equation which determines the chemical potential $\mu$ is described as

$$
\begin{aligned}
n-n_{\mathrm{f}}\left(\mu, T_{\mathrm{c}}\right) & =2 n_{\mathrm{b}}\left(\mu, T_{\mathrm{c}}\right) \\
& =\frac{1}{N_{\mathrm{L}}} \sum_{\boldsymbol{q}_{\tau}} g\left(\eta \boldsymbol{q}_{\tau}\right),
\end{aligned}
$$

where $\eta_{\boldsymbol{q}_{ \pm}}$is a discrete pole of $\left[1-\chi_{ \pm}^{d d}(\boldsymbol{q}, \omega)\right]^{-1}$ and $g(\omega)$ represents the Bose distribution function. In order to subsequently define the Fermi energy, we introduce $\mu^{*}$ as $n_{\mathrm{f}}\left(\mu^{*}, T_{\mathrm{c}}\right)=n$ for a given total density $n$. Equation (8) is called Thouless criterion, which results in the conventional BCS gap equation and gives the superconducting transition temperature evaluated in the meanfield thory $T_{\mathrm{c}}^{\mathrm{MF}}$ if we neglect $n_{\mathrm{b}}$ and substitute $n=n_{\mathrm{f}}$ in eq. (9). The summation of momentum and layer in eq. (9) is executed, except for the point which satisfies eq. (8), in order to remove the divergence.

\section{EPS File fig2.ps not found}

Fig. 2. $U_{d}$-dependence of $T_{\mathrm{c}}^{\mathrm{NSR}}$ and $T_{\mathrm{c}}^{\mathrm{MF}}$ for $n=4.80$. The solid line and the dashed one denote $T_{\mathrm{c}}^{\mathrm{NSR}}$ and $T_{\mathrm{c}}^{\mathrm{MF}}$ respectively, and both are for $t_{z}=0.00 t$ and $\varepsilon_{d}=1.20 t$. We obtain these results by the interpolation based on the $n$-dependence of $T_{\mathrm{c}}$ for every $U_{d}$.

In the numerical calculation, we set $\varepsilon_{p} \equiv 0$ and control the filling by shifting $\mu$. We define the Fermi energy $E_{\mathrm{F}}$ as follows:

$$
\begin{gathered}
E_{\mathrm{F}} \equiv \mu^{*}-E_{\mathrm{F}}^{0}, \\
n_{\mathrm{f}}\left(E_{\mathrm{F}}^{0}, 0\right)=4.0,
\end{gathered}
$$


which means that $E_{\mathrm{F}}=0$ i.e. $\mu^{*}=E_{\mathrm{F}}^{0}$ when the highest band is empty. FBZ is divided into $64 \times 64$ mesh, and the other input parameters are set like, $\varepsilon_{d}=0.40 t, 0.80 t$, $1.20 t, t_{p p}=0.40 t, U_{d}=4.0 t, t_{z}=0.00 t$ for monolayer case or $t_{z}=0.20 t$ for bilayer case. When we numerically integrate the sum in eq. (9), we introduce the cutoff momentum $q_{\mathrm{c}}^{ \pm}=(5 / 32) \pi$ assuming weak 3-dimensionality of real systems, in order to avoid the large fluctuation which is unique to lower-dimensional system. In practice, first we investigate $T_{c}^{\mathrm{MF}}$ for a given $\mu$, then calculate $n_{\mathrm{b}}\left(\mu, T_{\mathrm{c}}\right)$ and determine $\mu^{*}$ for the total density $n$. Thus, the $n_{\mathrm{f}}\left(\mu^{*}\right) \equiv n$ to $T_{\mathrm{c}}^{\mathrm{NSR}}$ curve and the $n_{\mathrm{f}}(\mu) \equiv n$ to $T_{\mathrm{c}}^{\mathrm{MF}}$ curve can be obtained. Figure 1 shows these for the two differnt values of $t_{z}$. The difference between $T_{\mathrm{c}}^{\mathrm{MF}}$ and $T_{\mathrm{c}}^{\mathrm{NSR}}$ increases much near the half-filled state, which corresponds to $n=5.0$ for our model. While $T_{\mathrm{c}}^{\mathrm{MF}}$ are higher for larger $\varepsilon_{d}, T_{\mathrm{c}}^{\mathrm{NSR}}$ take almost the same values near the half-filling, independent of $\varepsilon_{d}$. It means that while $T_{\mathrm{c}}^{\mathrm{MF}}$ are determined by $U_{d} / W, T_{\mathrm{c}}^{\mathrm{NSR}}$ are determined only by the filling $n$, where $W$ is the bandwidth of the highest band, which decreases as $\varepsilon_{d}$ increases. In addition, the suppression of $T_{\mathrm{c}}^{\mathrm{NSR}}$ is sensitive to the exsistence of the interlayer transfer $t_{z}$, which is supposed to introduce the higher dimensionality and weaken the strong thermal fluctuation. In Fig. 2 we compare the $U_{d}$-dependence of $T_{\mathrm{c}}^{\mathrm{NSR}}$ and $T_{\mathrm{c}}^{\mathrm{MF}}$ for $n=4.80, t_{z}=0.00 t$ and $\varepsilon_{d}=1.20 t$. While $T_{\mathrm{c}}^{\mathrm{MF}}$ increases rapidly with $U_{d}, T_{\mathrm{c}}^{\mathrm{NSR}}$ is almost saturated in the strong coupling regime. For references, in Fig. 3 we roughly show the momentum dependence of $\eta_{\boldsymbol{q} \pm}$, which corresponds to the dispersion of a bosonic excitation i.e. a noncondensed Cooper pair, for two different fillings. $\eta_{\boldsymbol{q} \pm}$ becomes lower as $n$ approaches 5.0, and we expect that the densities of the noncondensed Cooper pairs are increased and that the Bose condensation temperatures i.e. $T_{\mathrm{c}}^{\mathrm{NSR}}$ of the pairs are much suppressed, compared with the temperature where the Cooper instability occurs i.e. $T_{\mathrm{c}}^{\mathrm{MF}}$. In Fig. 4, we show the relation between $T_{\mathrm{c}}^{\mathrm{NSR}} / E_{\mathrm{F}}$ and $E_{\mathrm{F}}$ for each set of parameters. In our calculation, for every case $T_{\mathrm{c}}^{\mathrm{NSR}} / E_{\mathrm{F}}$ shows a maximum in the intermediate coupling regime. This result is an artifact of approximations, and the method to remove such a maximum has been already discussed by R. Haussmann in his paper.14 However, in the strong coupling regime, all qualitative results are consistent independent of any kinds of approximations, and we find that $T_{\mathrm{c}}^{\mathrm{NSR}} / E_{\mathrm{F}}$ tend to settle with constant values.

In summary, we have calculated $T_{\mathrm{c}}^{\mathrm{NSR}}$ of the bilayer $d$ - $p$ model on the assumption that $d_{x^{2}-y^{2}}$-wavelike attractive intraction works on $d$-electrons. Compared with $T_{\mathrm{c}}^{\mathrm{MF}}, T_{\mathrm{c}}^{\mathrm{NSR}}$ are much suppressed in the strong coupling regime. This effect is due to the thermal fluctuation of preformed Cooper pairs and can be weakened by the exsistence of the interlayer coupling. Near the half-filled state, $T_{\mathrm{c}}^{\mathrm{NSR}}$ is almost propotional to Fermi energy $E_{\mathrm{F}}$. However, our model does not include the on-site repulsive interaction among $d$-electrons, which is very important to explain the Mott-insulating state near half-filling. In real systems, $E_{\mathrm{F}}$ should be much reduced near halffilling by this strong correlation, where $T_{\mathrm{c}}$ decreases inspite of strong coupling. Therefore, if we include the strong on-site repulsion by an appropriate method and
EPS File fig3.ps not found

Fig. 3. $\boldsymbol{q}$-dependence of $\eta_{\boldsymbol{q} \pm}$ near $(0,0)$ for the two different fillings. These data have been reconstructed from the real numerical data by an appropriate method so that their general view can be easily observed. The solid line and the dashed one denote for $n=4.1528, T_{\mathrm{c}}^{\mathrm{NSR}}=0.01296 t$ and for $n=4.7198$, $T_{\mathrm{c}}^{\mathrm{NSR}}=0.12625 t$, respectively. Both are for $t_{z}=0.00 t$ and $\varepsilon_{d}=1.20 t$. Arrows show the points for $\boldsymbol{q}=\left(q_{\mathrm{c}}, 0\right)$ and $\boldsymbol{q}=\left(0, q_{\mathrm{c}}\right)$.

EPS File fig4.ps not found

Fig. 4. The ratios of $T_{\mathrm{c}}\left(\equiv T_{\mathrm{c}}^{\mathrm{NSR}}\right)$ to Fermi energy $E_{F}$ in (a) for $t_{z}=0.00 t$ and in (b) for $t_{z}=0.20 t$ as functions of $E_{F} / t$. In both panels the open circles, squares and diamonds correspond to $\varepsilon_{d}=0.40 t, 0.80 t$ and $1.20 t$, respectively.

renormalize $E_{\mathrm{F}}$ of the quasiparticle, we might obtain a more realistic picture of the underdoped cuprates. Finally, the recent experiment has clarified that the reduced gap $2 \Delta_{0} / k_{\mathrm{B}} T^{*}$ is nearly constant for the underdoped $\mathrm{Bi}_{2} \mathrm{Sr}_{2} \mathrm{CaCu}_{2} \mathrm{O}_{8+\delta}$, where $2 \Delta_{0}$ is the magnitude of the superconducting gap estimated by STS and $T^{*}$ is the temperature at which the in-plane resistivity starts to deviate from the $T$-linear behavior.15) This result suggests the close relationship between $T^{*}$ and preformed incoherent pairs. Consequently, our future problem is to obtain $2 \Delta_{0}$ consistently and study the correlation between $2 \Delta_{0}$ and $T^{*}\left(\simeq T_{\mathrm{c}}^{\mathrm{MF}}\right)$ or $T_{\mathrm{c}}\left(\simeq T_{\mathrm{c}}^{\mathrm{NSR}}\right)$ by the use of more realistic interaction among $d$-electrons in order to explain such experimental results.

The authors are grateful to Prof. M. Ido, Prof. M. Oda, Prof. T. Takahashi and Dr. T. Yokoya for sharing recent experimental results and fruitful discussions. One of the authors (S. K.) is grateful to Dr. T. Hotta for his useful comments and discussions. They also thank the Supercomputer Center, Kyoto University and the Supercomputer Center, Institute for Solid State Physics, University of Tokyo, for the facilities and the use of the FACOM VPP500. This work has been supported by a Grant-in-Aid for Scientific Research on Priority Areas "Anomalous Metallic State near the Mott Transition" from the Ministry of Education, Science, Sports and Culture, Japan.

[1] B. Batlogg, H. Y. Hwang, H. Takagi, R. J. Cava, H. L. Kao and J. Kwo: Physica C 235-240 (1994) 130.

[2] J. W. Loram, K. A. Mirza, J. M. Wade, J. R. Cooper and W. 
Y. Liang: Physica C 235-240 (1994) 134.

[3] G. V. M. Williams, J. L. Tallon, R. Michalak and R. Dupree: Phys. Rev. B 54 (1996) R6909.

[4] Y. Itoh, T. Machi, A, Fukuoka, K. Tanabe and H. Yasuoka: J. Phys. Soc. Jpn. 65 (1996) 3751.

[5] A. V. Puchkov, P. Fournier, D. N. Basov, T. Timusk, A. Kapitulnik and N. N. Kolesnikov: Phys. Rev. Lett. 77 (1996) 3212.

[6] T. Tanamoto, H. Kohno and H. Fukuyama: J. Phys. Soc. Jpn. 63 (1994) 2739.

[7] H. Ding, T. Yokoya, J. C. Campuzano, T. Takahashi, M. Randeria, M. R. Norman, T. Mochiku, K. Kadowaki and J. Giapintzakis: Nature 382 (1996) 51; H. Ding, M. R. Norman, T. Yokoya, T. Takeuchi, M. Randeria, J. C. Campuzano, T. Takahashi, T. Mochiku and K. Kadowaki: Phys. Rev. Lett. 78 (1997) 2628.

[8] A. G. Loeser, Z. -X. Shen, D. S. Dessau, D. S. Marshall, C. H. Park, P. Fournier and A. Kapitulnik: Science 273 (1996) 325; D. S. Marshall, D. S. Dessau, A. G. Loeser, C. H. Park, A. Y. Matsuura, J. N. Eckstein, I. Bozovic, P. Fournier, A. Kapitulnik, W. E. Spicer and Z. -X. Shen: Phys. Rev. Lett. 76 (1996) 4841.

[9] P. Nozières and S. Schmitt-Rink: J. Low. Temp. Phys. 59 (1985) 195.

[10] S. Schmitt-Rink, C. M. Varma and A. E. Ruckenstein: Phys. Rev. Lett. 63 (1989) 445.

[11] A. Tokumitu, K. Miyake and K. Yamada: Prog. Theor. Phys. Supp. 106 (1991) 63; A. Tokumitu and K. Miyake: Physica C 235-240 (1994) 2367.

[12] C. A. R. Sá de Melo, M. Randeria and J. R. Engelbrecht: Phys. Rev. Lett. 71 (1993) 3202.

[13] S. Yoda and K. Yamada: J. Phys. Soc. Jpn. 66 (1997) 1398.

[14] R. Haussmann: Phys. Rev. B 49 (1994) 12975.

[15] M. Oda, K. Hoya, R. Kubota, C. Manabe, N. Momono, T. Nakano and M. Ido: Physica C 281 (1997) 135. 


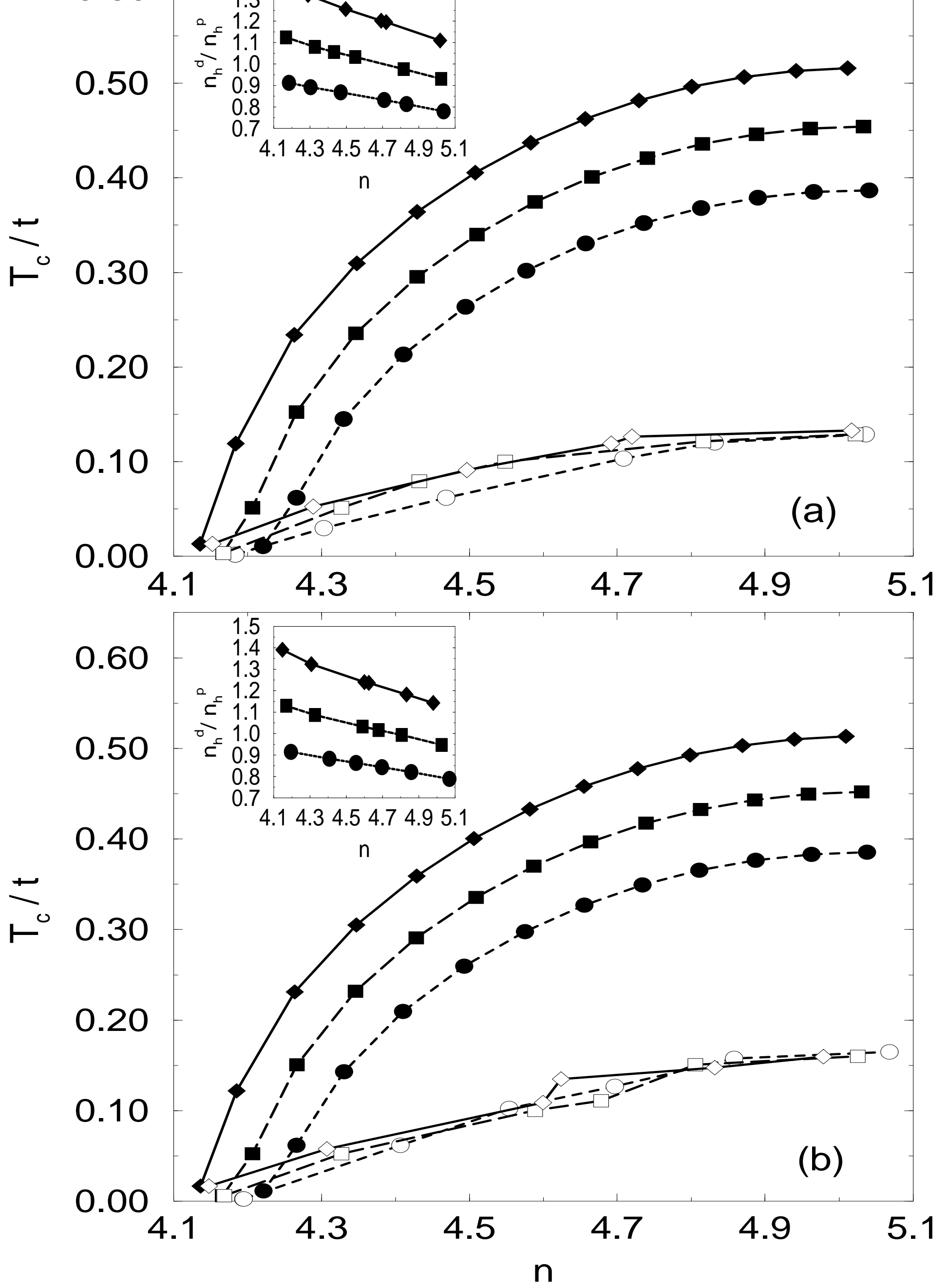




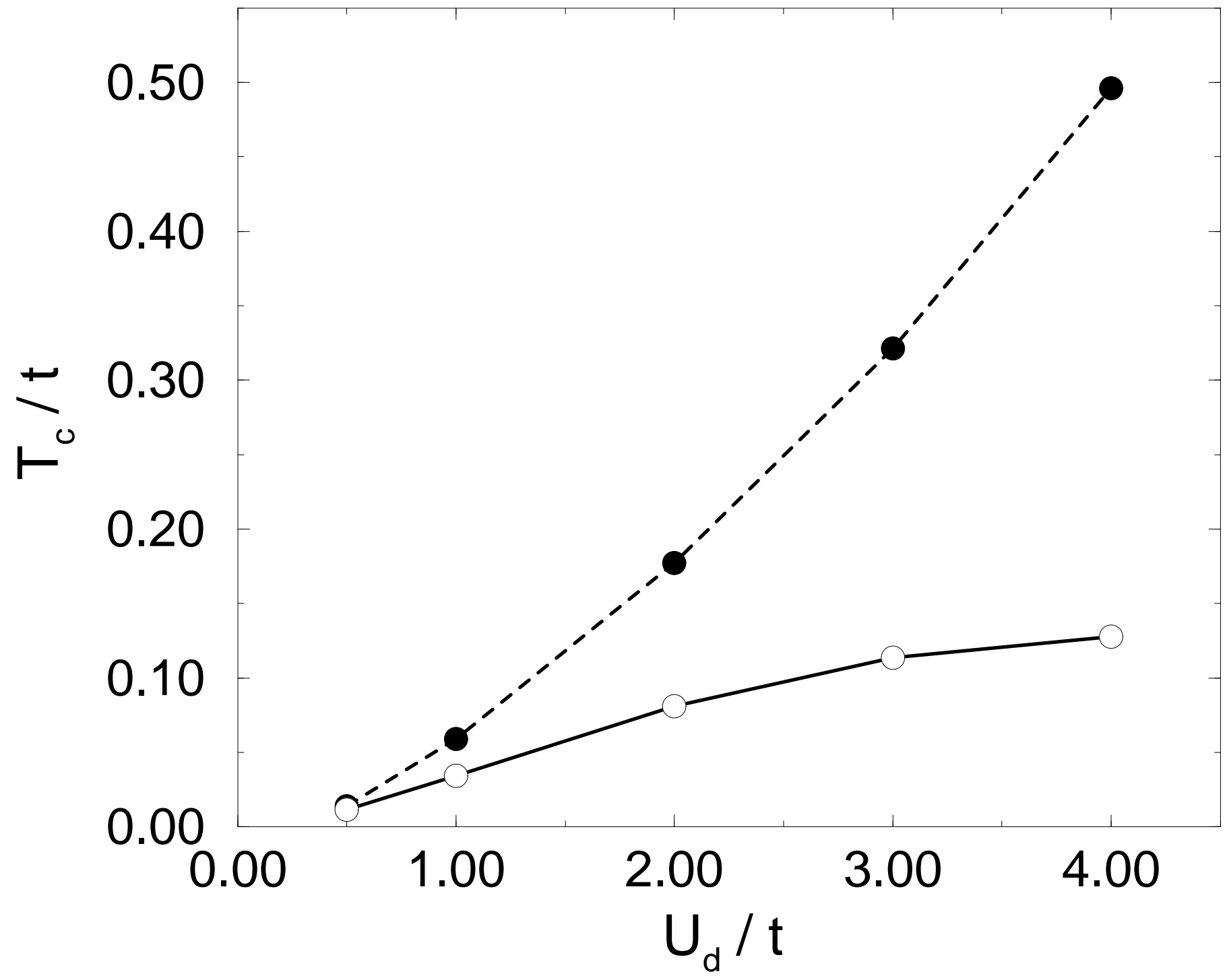

